-1889.] Physical Training of the Insane.

A personal knowledge of gymnastics by the medical officers is almost essential to their successful introduction.into institutions it seems to me, or at any rate such a knowledge very much enhances the chances of success, as they cannot otherwise as intelligently supervise or direct their application.

In the use of gymnastics for the insane, the subject is less able than in the community at large to give any evidence of whether the work is too hard for him, or not suited to him, and hence it is especially necessary to understand both the changes produced in him by mental disease, and the general effect of gymnastic exercises on persons in health.

\title{
REMARKS ON PHYSICAL TRAINING OF THE INSANE.*
}

BY MISS A. W. ADAMS, Instructor in Gymnastics at Dr. Channing ${ }^{\circ}$ Private Hospital.

Dr. Channing has mentioned the exercises which we shall illustrate here this afternoon, which I will explain more fully. Our system induces no thought on the part of the pupil, nor real mental effort. The evil we are trying to counteract is the abnormal pressure, consequently pupils work entirely by imitation, which is largely intuitive, and little call is made on the nerve force. Ilerein we differ materially from the present promulgators of the ling system, though all systems of harmonious bodily development are based upon the thought of Ling.

This principle makes our system particularly adaptable to the insane and nerrous, as the object is to send through the system the purest blood, with the least possible exhaustion of nerve force. The work is divided into yearly courses, and is carried on in a slow but progressve manner, commencing with simple elementary work-one movement dependent upon and leading up to the next, until finally the whole body is exercised without apparent effort on the part of the pupil.

I have endeavored to carry out these same methods with the insane-with the exception that I am obliged to go much more slowly, often to omit alternate movements and complicated ones entirely.

* Being part of the discussion which followed the reading of Dr. Channing's paper. 
Instead of giving three or four rests during the bour as is customary with beginners, $I$ find it is better to keep the class working steadily for thirty or forty minutes and then dismiss them. By so doing I held their attention better and kept the interest up. With very nervous, delicate patients who are not strong enough to take the exercises standing, very slow, stretching movements on the back are given, which have a very soothing, quieting effect.

I open with free movements given for the purpose of starting the circulation, limbering the joints, and exercising a large number of the muscles greatly from head to foot. The general order for the series being movements for the head, shoulders, whole arm, forearm, wrist, fingers, trunk, whole leg, lower leg and ankle.

We next pass to the side pull, the elementary work at the chest weights, using the right and left arms alternately. This series develops the pectoralis major, lattissimus dorsi, trapezius, biceps, and all the grip muscles of the arms. The double chest weights is more advanced work, in which both hands are used at the same time and exercise principally the biceps, triceps, lattissimus dorsi, deitoid, pectorals and stretches back from neck to heel and gives a churning movement to the abdomen.

The bells used are very light, in order that the body or chest may be exercised as well as the arms. The series with both wooden and iron dumb bells, also wands, are combined with the object of exercising not only the muscles of the arm, chest and back, but of the leg, by giving stooping and bending movements.

In the iron bells the movements are very slow and gliding, giving more of a sustained action to the muscle, while in the wooden the active movement and rest is much more marked. During the period of exercise, the movements increase in force, so that at the end of the hour the pupil leaves the floor, in a brisk glow, ready for a bath. The closing exercises are either a quick march or breathing movements, the latter having a more quieting effect upon the insane. 